\title{
Reliability Analysis of Common Cause Failure Multistate System Based on CUGF
}

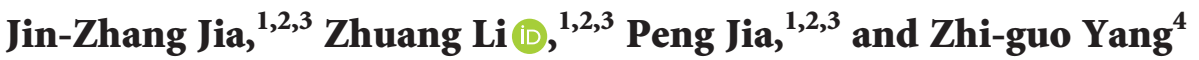 \\ ${ }^{1}$ College of Safety Science and Engineering, Liaoning Technical University, Fuxin 123000, China \\ ${ }^{2}$ Institute of Safety Science, Liaoning Technical University, Fuxin 123000, China \\ ${ }^{3}$ Key Laboratory of Mine Thermo-motive Disaster and Prevention, Ministry of Education, Fu Xin123000, China \\ ${ }^{4}$ Liaohe Oilfield Construction Co., Ltd., Panjin 124000, China \\ Correspondence should be addressed to Zhuang Li; leezhg@126.com
}

Received 14 November 2019; Revised 19 March 2020; Accepted 16 April 2020; Published 22 May 2020

Academic Editor: Elena Zaitseva

Copyright ( 92020 Jin-zhang Jia et al. This is an open access article distributed under the Creative Commons Attribution License, which permits unrestricted use, distribution, and reproduction in any medium, provided the original work is properly cited.

\begin{abstract}
This paper addresses the problem of mixed uncertainty in the reliability analysis of multistate systems under common cause failure conditions. Combining the cloud model theory, universal generation function (UGF) method, and common cause failure theory, the universal generation function method is extended based on a probabilistic cloud model, i.e., the cloud universal generation function (CUGF) analysis method. The cloud model represents the random and cognitive uncertainty of the state probability, i.e., mixed uncertainty. Next, through CUGF, according to the calculation rules of cloud operators, we provide steps to obtain the reliability of a multistate system under independent failure and common cause failure conditions and obtain cloud digital features for reliability. The accuracy and feasibility of the method are verified by a numerical example. This paper solves the problem of reliability analysis of multistate systems with mixed uncertainty in unit state probability information under common cause failure conditions. We integrate system multistate, information uncertainty, and common cause failure for reliability analysis to avoid large errors, more in line with a project's actual situation. We propose new ideas and methods to process randomness and fuzzy information or data in multistate system reliability analysis.
\end{abstract}

\section{Introduction}

Traditional system reliability analysis generally assumes that the state of a system and unit is normal or fully failed. This is often referred to as a two-state system. Due to the complexity of the system structure, environmental factors, working conditions, and efficiency changes, reliability analysis using a two-state correlation theory system is no longer applicable in practical engineering. There are several transitional states of a system and unit between normal and complete failures, and each exists with a certain probability. A multistate system (MSS) generally refers to a situation in which complex systems and units can have different states, characterized by different levels of performance. MSS research has entered the mainstream. In the 1970s, Barton [1], Murchland [2], Barlow and Wu [3], and El-Neveihi [4] successively proposed and improved the concept and related theory of MSS. Since the introduction of MSS reliability theory, many results have been produced that are widely used in fields including machinery [5-8], electric power [9-12], aerospace [13-15], and networks [16-19]. Reliability analysis of an MSS is more complicated than that of two-state systems. Lisnianski and Levitin [20] studied MSS reliability theory and analytical methods in application. There are four main methods for MSS reliability analysis and evaluation: the structural function method [20-23], stochastic process method [24-26], Monte Carlo simulation [27-29], and universal generative function (UGF) method [20, 30-32]. The structural function method, stochastic process, and Monte Carlo simulation for complex large-scale MSS analysis are time-consuming and can result in a "state dimension explosion" in the calculation process. UGF can reduce computational complexity, improve efficiency, and quickly solve the problem of large-scale, complex MSS reliability analysis. Ushakov [33,34] proposed UGF, and Lisnianski [20] and 
Levitin [30] introduced the UGF method to the field of MSS reliability, where it has been widely used. The classical UGF method is based on two hypotheses [15, 35]: (1) each state probability of a subsystem or unit of an MSS can be accurately measured by probability; and (2) the performance status of a subsystem or unit is accurate.

In engineering practice, due to the complexity of the system structure and the insufficient number of test samples, accurate values of the state performance level and state probability of each component are difficult to obtain, but upper and lower limits of the unit state probability are easier to obtain. Therefore, the probability-based method is no longer applicable. Nonprobabilistic methods such as fuzzy theory [36], interval theory [37], and evidence theory $[38,39]$ have been successively proposed and developed and gradually became means of MSS reliability analysis under uncertainty. Uncertainty can be categorized as either random or cognitive [40], the former consisting of variability and randomness of a unit and the latter caused by incomplete and inaccurate knowledge or data [41]. Scholars have promoted the classic UGF method according to the above nonprobabilistic methods. Ding $[35,42]$ defined a fuzzy MSS system. Given fuzzy state performance and fuzzy state probability, the fuzzy general generation function was proposed to analyze and evaluate fuzzy MSS reliability. Based on interval theory, Li [43] converted the fuzzy numbers to interval numbers and used the interval general generation function to evaluate the reliability of an interval MSS system. Destercke and Sallak [44] applied the evidence generic generation function to extend UGF to assess the reliability of an MSS. However, these methods have certain limitations regarding the reliability analysis of an MSS with mixed uncertainty information. The fuzzy UGF method can easily lose part of the information in a subsystem or unit, and the calculation process is complicated and costly [45]. The interval expansion and overevaluation of the interval UGF method limit the accuracy of reliability. The evidence UGF cannot determine MSS reliability from fuzzy information. MSS unit status probability information and performance levels are mainly derived from historical data, test data, expert experience, and field-use data. These data have mixed problems of randomness and fuzziness. It is difficult to accurately analyze mixed uncertainty information with these methods. The application cloud model can represent the mixed uncertainty of state probability and performance level information of each unit of the MSS [45]. Deyi [46, 47] proposed a cloud theory whose core was a cloud model, combining random and fuzzy characteristics. Reliability analysis using a cloud model solves mixed uncertainty problems such as random and fuzzy MSS information [47]. Reliability data in engineering practice are often random and fuzzy. A cloud model represents the uncertainty of unit or subsystem state probability and state performance information, and the cloud universal generating function (CUGF) and MSS reliability analysis are more in line with a project's actual situation.

When analyzing MSS reliability, failures between units are usually assumed to be independent, but this often does not reflect real engineering situations. Failures between units are not independent, but there is related failure. Complex MSS ignores related failures, and the calculated results produce certain errors. Common cause failure is a common form of related failure in which two or more units fail together for common external or internal reasons, and, regarding the failure of MSS common cause, failure has been studied by both explicit and implicit methods. The former are usually used when the cause of failure is obvious and can be incorporated in the fault tree model as an independent basic event, and implicit modeling is used when the cause is more complicated. Levitin [48] and Liyang [49] used the general generation function to analyze the reliability of an irreparable MSS with common cause failure. Levitin [50] analyzed common cause failures in common source failures. Jinyu and Liyang [51] discussed the mechanism of common cause failure and its effect. Based on the load discretization method and UGF, a quantitative evaluation model of MSS reliability was established. Chunyang [52] established and optimized an MSS reliability model considering common cause failure. Mi [53] combined common cause failure and the evidence general generation function to analyze the reliability of an MSS with uncertain information. The common cause probability reflects the complexity of the MSS structure, subjectivity of expert experience, and errors in the test data. The above analysis does not consider the uncertainty of the common cause failure probability. Information uncertainty and related failure will be a primary direction of future complex system reliability research. This paper combines the uncertainty of MSS unit state probability information and common cause failure information uncertainty in reliability analysis.

The rest of this paper is organized as follows. Section 2 introduces the cloud model used to represent the theoretical knowledge of information uncertainty. Section 3 reviews the traditional UGF and CUGF modeling methods. Section 4 introduces the CUGF modeling method due to the uncertainty of the failure probability and the common cause failure condition. In Section 5, we provide the example of a series-parallel structure MSS system of fluid transmission; we compare and analyze the reliability of MSS under independent interruption and common cause failure. In Section 6, we provide our conclusions and discuss future prospects.

\section{Cloud Model}

\subsection{Definition and Digital Characteristics of the Cloud}

2.1.1. Definition of Cloud. The set quantity domain $U$ is represented by an exact value. The qualitative concept on $U$ is $C$. The quantitative value $x \in U$ has a stable tendency random number $y=\mu_{C}(x)$, and $C$ has a membership degree of $x$. The distribution of the membership degree $y$ on the domain $U$ is called the subordinate cloud, referred to as the "cloud." The cloud is composed of a large number of cloud droplets, and each $(x, y)$ is called a cloud drop. The generation process of cloud droplets represents a mutual mapping between qualitative and quantitative concepts and is a quantitative description of qualitative concepts. The 
cloud is the mapping of the domain $X$ to the interval $[0,1]$ [47].

2.1.2. Digital Characteristics of the Cloud. The expected value $E x$, entropy $E n$, and super entropy $H e$ are numeric characteristic parameter values of the cloud. Ex is the central value of the domain, the most representative qualitative concept point. En is a qualitative measure of uncertainty, reflecting the randomness and fuzziness of a concept and its correlation [45]. The larger the En, the greater the degree of blur; $\mathrm{He}$ is hyperentropy, which reflects the uncertainty of entropy $E n$, as determined by the randomness and fuzziness of entropy. The larger the He, the thicker the cloud and the greater the degree of dispersion.

2.2. Cloud Generator. The normal distribution in probability theory and the bell-shaped membership function in fuzzy set theory are the most common and expressive distribution functions. The cloud model developed on the basis of the two is called the normal cloud model. We use the normal cloud model, which is universal, to represent the uncertainty of unit state probability information [46].

2.2.1. Positive Normal Cloud Generator. The forward cloud generator is a process of generating cloud drops from digital feature parameter values to form a cloud, as shown in Figure 1. The digital characteristic parameters $(E x, E n, H e)$ are input, and a normal cloud of $m$ cloud droplets $\left(x_{l}, \mu_{l}\right)$ $(l=1,2, \ldots, m)$ is output as follows:

(1) Generate $m$ normal random numbers $E n_{l}^{\prime}=N(E n, H e)$, where $N(E n, H e)$ denotes a normal distribution

(2) Generate $m$ normal random numbers $x_{l}=N\left(E x, E n_{l}^{\prime}\right)$, where $N\left(E x, E n_{l}^{\prime}\right)$ denotes a normal distribution

(3) Generate the membership degree $\mu_{l}=\exp \left[-\left(x_{l}-E x\right)^{2} /\left(2 E n_{l}^{\prime 2}\right)\right]$, where $x_{l}$ is the normal random number generated in step 2

2.2.2. Reverse Normal Cloud Generator. The reverse normal cloud generator obtains the numeric characteristic parameter values of the cloud according to a certain number of normally distributed cloud drops, as shown in Figure 2. We input $m$ sample points $x_{l}(l=1,2, \ldots, m)$, and digital characteristic parameters $(E x, E n, H e)$ are output. The inverse normal cloud generator algorithm is based on statistical principles and is divided into containing certainty information and not containing certainty information. This paper uses the algorithm proposed by Liu et al. [54], which contains no certainty information. The steps are as follows:

(1) Calculate the mean of the sample $\bar{X}=(1 / m) \sum_{l=1}^{m} x_{l}$, the absolute center distance of the first-order sample $M=(1 / m) \sum_{l=1}^{m}\left|x_{l}-x\right|$, and the variance of the sample $S^{2}=(1 / m-1) \sum_{l=1}^{m}\left(x_{l}-\bar{x}\right)^{2}$

(2) $E x=\bar{X}$

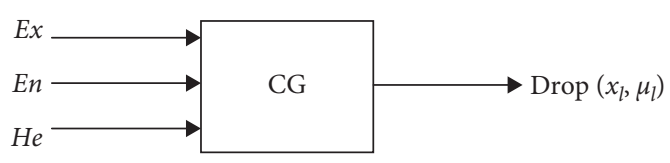

FIGURE 1: Forward generator.

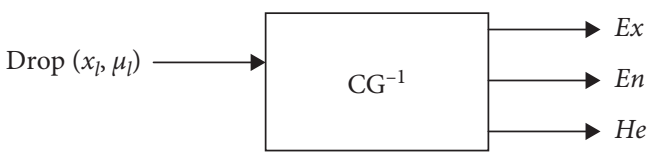

Figure 2: Reverse generator.

(3) $E n=\sqrt{\pi / 2} \times M$

(4) $\mathrm{He}=\sqrt{S^{2}-E n^{2}}$

\section{Cloud Generic Generating Function}

3.1. Classic Generic Generating Function. Classical UGF theory is aimed at discrete random variables and is embodied as a single variable in UGF. The distribution law of discrete random variables consists of two sets: $G$ represents the state performance of the system, and $P$ represents the corresponding probability. For unit $G^{j}$ of the MSS, if the state $g^{j}$ of each unit and the probability $p^{j}$ corresponding to the state are given, then the UGF form of the unit can be obtained as

$$
U_{j}(Z)=\sum_{i=1}^{k_{j}} p_{i}^{j} z^{g_{i}^{j}},
$$

where $k_{j}$ is the state of the $j$ th unit. The MSS consisting of these units can obtain the UGF form of the entire system as

$$
U(Z)=\Omega_{\phi}\left(U_{1}(Z), \ldots, U_{n}(Z)\right)=\sum_{i_{1}=1}^{k_{1}} \sum_{i_{2}=1}^{k_{2}} \ldots \sum_{i_{n}=1}^{k_{n}} \prod_{j=1}^{n} p_{i_{j}}^{j} Z^{\phi\left(g_{i_{1}}^{1}, \ldots, g_{i_{n}}^{n}\right)},
$$

where $\Omega_{\varphi}$ is a combined operator determined by the system structure function depending on $Z$. For a series structure, the operator is the minimum function

$$
\phi_{P}\left(g_{i_{1}}^{1}, g_{i_{2}}^{2}, \ldots, g_{i_{n}}^{n}\right)=\min \left\{g_{i_{1}}^{1}, g_{i_{2}}^{2}, \ldots, g_{i_{n}}^{n}\right\} .
$$

For a parallel structure, the operator is the sum function:

$$
\phi_{S}\left(g_{i_{1}}^{1}, g_{i_{2}}^{2}, \ldots, g_{i_{n}}^{n}\right)=\sum_{j=1}^{n} g_{i_{n}}^{n} .
$$

When the system state satisfies $r_{i}=g_{i}-w \geq 0$, then the state $i$ is acceptable, and $w$ is the system requirement. The reliability of the MSS can generally be defined as the probability whose performance level is greater than the demand:

$$
R(w)=\sum_{r_{i \geq 0}} p_{i} .
$$

For a given $U(Z)$ and $w$, the steady-state reliability of the MSS can be obtained by applying the operator $\delta_{R}$; i.e., 


$$
\begin{aligned}
R(w) & =\delta_{A}(U(Z), w)=\delta_{R}\left(\sum_{i=1}^{K} p_{i} Z^{g_{i}}, w\right)=\sum_{i=1}^{K} p_{i} \alpha_{i}, \\
\alpha_{i} & = \begin{cases}1, & r_{i} \geq 0 \\
0, & r_{i}<0 .\end{cases}
\end{aligned}
$$

3.2. Cloud Generic Generating Function. The information of the state probability of each unit of an MSS mainly comes from historical data, test data, physical characteristics, expert experience, and experience in use. The more accurate and sufficient the information is, the closer it is to actual values. However, historical data, experimental data, and other quantitative data in actual engineering are subject to experimental errors and inaccurate field monitoring. Qualitative information such as expert experience and use experience is subject to human judgment, so there are random uncertainty (randomness) and cognitive uncertainty (fuzziness). The cloud model is used to represent the mixed uncertainty of the state probability information or data of each unit of the MSS. The obtained state probability cloud contains mixed uncertainty, and the probability cloud model is combined with UGF to obtain the CUGF method. Using the calculation rules of CUGF and cloud operators, the numeric characteristic parameter values of MSS reliability under given requirements are obtained.

Assume that an MSS is composed of $n$ units, each unit has $k$ states, and much information or data constitutes a sample of the unit state probability. These data include historical data, test data, expert experience, and usage experience. The information consists of quantitative data and qualitative information. $m$ quantitative data points constitute a quantitative sample set $X=\left\{x_{1}, x_{2}, \ldots, x_{m}\right\}$, and $q$ items of qualitative information constitute a qualitative sample set $Y=\left\{y_{1}, y_{2}, \ldots\right.$, $\left.y_{q}\right\}$. The distribution of samples is a probability cloud of unit states, which contains the random and cognitive uncertainty of the state probability. The process is as follows:

(1) Suppose that the state probability of unit $j$ is derived from historical, experimental, and quantitative data. For the composed sample set $X=\left\{x_{1}, x_{2}, \ldots, x_{m}\right\}$, according to the algorithm in Section 2.2.2, the data in sample $X$ are substituted in the inverse normal cloud generator, and the mean value $\square X$, center distance $M$, and variance $S^{2}$ are obtained, so as to obtain the numerical characteristic parameter values of the probability cloud $C p_{i_{j}}^{j}$, and recorded as $C p_{i_{j}}^{j}(E x, E n$, $\mathrm{He}$ ). According to the algorithm in Section 2.2.1, the digital characteristic parameter values to be obtained are substituted in the forward normal cloud generator, the membership degree $\mu_{l}$ of $x_{l}$ can be obtained, and the cloud drops $\left(x_{l}, \mu_{l}\right)$ of the probability cloud $C p_{i}^{j}$ $(E x, E n, H e)$ are generated. A probabilistic normal cloud map is formed, reflecting the randomness and fuzziness of the unit state probability.
(2) The qualitative information from which the state probability of unit $j^{\prime}$ is derived from is often described in natural language. Each expert understands the digital characteristic parameter values of empirical settings based on his or her experience. The state probability of information sample $Y$ composed of $q$ experts is calculated according to formula (8) $[55,56]$, and the numeric characteristic parameter values of the unit state probability cloud $C p_{i_{j}}^{j}$ are obtained and recorded as $C p_{i_{j}}^{j}\left(E x^{\prime}, E n^{\prime}, H e^{\prime}\right)$ :

$$
\left\{\begin{array}{l}
E^{\prime} x=\frac{\left(E^{\prime} x_{1} \times E^{\prime} n_{1}+E^{\prime} x_{2} \times E^{\prime} n_{2}+\ldots+E^{\prime} x_{q} \times E^{\prime} n_{q}\right)}{\left(E^{\prime} n_{1}+E^{\prime} n_{2}+\ldots+E^{\prime} n_{q}\right)} \\
E^{\prime} n=E^{\prime} n_{1}+E^{\prime} n_{2}+\ldots+E^{\prime} n_{q} \\
H^{\prime} e=\frac{H^{\prime} e_{1} \times E^{\prime} n_{1}+H^{\prime} e_{2} \times E^{\prime} n_{2}+\ldots+H^{\prime} e_{q} \times E^{\prime} n_{q}}{\left(E^{\prime} n_{1}+E^{\prime} n_{2}+\ldots+E^{\prime} n_{q}\right)}
\end{array}\right.
$$

According to the algorithm in Section 2.2.1, the digital characteristic parameter values to be obtained are substituted in the forward normal cloud generator, the membership degree $\mu_{l}^{\prime}$ of $x_{l}^{\prime}$ can be obtained, and the cloud drops $\left(x_{l}^{\prime}, \mu_{l}^{\prime}\right)$ of the probability cloud $C p_{i_{j}}^{j}\left(E x^{\prime}, E n^{\prime}, H e^{\prime}\right)$ are generated. A probability normal cloud map is formed, which reflects the randomness and fuzziness of the unit state probability.

(3) The probability cloud of different states composed of quantitative data by unit $j$ is $C p_{i_{j}}^{j}(E x, E n, H e)=\left\{C p_{1_{j}}^{j}\right.$ $(E x, E n, H e), C p_{2_{j}}^{j}(E x, E n, H e), \ldots, C p_{k_{j}}^{j}(E x, E n$, $\mathrm{He})\}$.

When the probability cloud of unit $j$ is $C p_{i_{j}}^{j}(E x, E n, H e)$, then the CUGF of unit $j$ is expressed as

$$
U^{\prime j}(Z)=\sum_{j=1}^{K_{j}} C p_{i_{j}}^{j}(E x, E n, H e) Z^{g_{i j}^{j}} .
$$

(4) The probability cloud of different states composed of qualitative, information for unit $j^{\prime}$ is $C p_{i_{j}}^{j}\left(E x^{\prime}, E n^{\prime}\right.$, $\left.H e^{\prime}\right)=\left\{C p_{1}^{j}\left(E x^{\prime}, E n^{\prime}, H e^{\prime}\right), C p_{2}^{j}\left(E x^{\prime}, E n^{\prime}, H e^{\prime}\right), \ldots\right.$, $\left.C p_{k_{j}}^{j}\left(E x^{\prime}, E n^{\prime}, H e^{\prime}\right)\right\}$

When the probability cloud of unit $j^{\prime}$ is $C p_{i_{j}}^{j}\left(E x^{\prime}, E n^{\prime}\right.$, $\left.H e^{\prime}\right)$, then its CUGF is

$$
U^{\prime j}(Z)=\sum_{j=1}^{K_{j}^{\prime}} C p_{i_{j}}^{\prime j}(E x, E n, H e) Z^{g_{i j}^{j}}
$$

(5) According to formulas (9) and (10), the CUGF of the entire MSS is 


$$
\begin{aligned}
U^{\prime}(Z)= & \Omega_{\varphi}\left(U^{1}(Z), \ldots, U^{n}(Z)\right)=\sum_{i_{1}=1}^{k_{1}} \sum_{i_{2}=1}^{k_{2}} \\
& \cdots \sum_{i_{n}=1}^{k_{n}} \prod_{j=1}^{n} C p_{i_{j}}^{j}(E x, E n, H e) \times Z^{\Phi\left(g_{i 1}^{1}, \ldots, g_{i n}^{n}\right)} \\
= & \sum_{i=1}^{k} C p_{i}(E x, E n, H e) Z^{g_{i}},
\end{aligned}
$$

where the composite operator $\Omega_{\varphi}$ in CUGF has the same meaning as the series-parallel structure of UGF.

MSS reliability analysis and evaluation involves operations between different state probability clouds $C p_{i_{j}}^{j}$. Cloud computing rules are shown in Table 1 [57].

In MSS reliability analysis, there are two basic operations, addition and multiplication, between state probability clouds $C p_{i_{j}}^{j}(E x, E n, H e)$. The product of each term in CUGF is solved according to the multiplication rule in Table 1 , and the numeric characteristic parameters of the probabilistic cloud are solved for. We determine the composite operator based on the MSS system relationship, the probabilistic cloud digital characteristic parameters of the same state are combined according to the addition rule in Table 1, and the probabilistic cloud characteristic parameter values of different states of MSS are obtained.

To explain the calculation rules of cloud operators, assume that an MSS is composed of two units, numbered 1 and 2. Each unit has two states $\{0,1\}$, and two units are connected in series. The probability cloud of state 0 of unit 1 is $C p_{0}^{1}=E x_{0}^{1}, E n_{0}^{1}, H e_{0}^{1}$, and the probability cloud of state 1 is $C p_{1}^{1}\left(E x_{1}^{1}, E n_{1}^{1}, H e_{1}^{1}\right)$. The probability cloud of state 0 of unit 2 is $C p_{0}^{2}\left(E x_{0}^{2}, E n_{0}^{2}, H e_{0}^{2}\right)$ and that of state 1 is $C p_{1}^{2}\left(E x_{1}^{2}, E n_{1}^{2}, H e_{1}^{2}\right)$. According to the cloud operator calculation rules, the detailed process is as follows.

(1) The CUGFs of units 1 and 2 are, respectively,

$$
\begin{aligned}
& U_{1}(Z)=C p_{0}^{1}\left(E x_{0}^{1}, E n_{0}^{1}, H e_{0}^{1}\right) Z^{0}+C p_{1}^{1}\left(E x_{1}^{1}, E n_{1}^{1}, H e_{1}^{1}\right) Z^{1}, \\
& U_{2}(Z)=C p_{0}^{2}\left(E x_{0}^{2}, E n_{0}^{2}, H e_{0}^{2}\right) Z^{0}+C p_{1}^{2}\left(E x_{1}^{2}, E n_{1}^{2}, H e_{1}^{2}\right) Z^{1} .
\end{aligned}
$$

(2) The cloud operator synthesis processes of MSS and CUGF are

$$
\begin{aligned}
U(Z)= & U 1(Z) U 2(Z)=\left[\left(E x_{0}^{1}, E n_{0}^{1}, H e_{0}^{1}\right) Z^{0}+\left(E x_{1}^{1}, E n_{1}^{1}, H e_{1}^{1}\right) Z^{1}\right] \\
& \cdot\left[\left(E x_{0}^{2}, E n_{0}^{2}, H e_{0}^{2}\right) Z^{0}+\left(E x_{1}^{2}, E n_{1}^{2}, H e_{1}^{2}\right) Z^{1}\right] \\
= & {\left[\left(E x_{0}^{1}, E n_{0}^{1}, H e_{0}^{1}\right)\left(E x_{0}^{2}, E n_{0}^{2}, H e_{0}^{2}\right)\right] Z^{0} } \\
& +\left[\left(E x_{0}^{1}, E n_{0}^{1}, H e_{0}^{1}\right)\left(E x_{1}^{2}, E n_{1}^{2}, H e_{1}^{2}\right)\right] Z^{\min (0,1)} \\
& +\left[\left(E x_{1}^{1}, E n_{1}^{1}, H e_{1}^{1}\right)\left(E x_{0}^{2}, E n_{0}^{2}, H e_{0}^{2}\right)\right] Z^{\min (1,0)} \\
& +\left[\left(E x_{1}^{1}, E n_{1}^{1}, H e_{1}^{1}\right)\left(E x_{1}^{2}, E n_{1}^{2}, H e_{1}^{2}\right)\right] Z^{\min (1,1)} .
\end{aligned}
$$

Let the calculation results of the digital characteristic parameters of the probabilistic cloud of the four states be
$\left(E x_{a}, E n_{a}, H e_{a}\right),\left(E x_{b}, E n_{b}, H e_{b}\right),\left(E x_{c}, E n_{c}, H e_{c}\right)$, and $\left(E x_{d}\right.$, $\left.E n_{d}, H e_{d}\right)$. The calculation for state $a$, for example, is

$$
\left\{\begin{array}{l}
E x_{a}=E x_{0}^{1} \times E x_{0}^{2}, \\
E n_{a}=\left|E x_{0}^{1} \times E x_{0}^{2}\right| \sqrt{\left(\frac{E n_{0}^{1}}{E x_{0}^{1}}\right)^{2}+\left(\frac{E n_{0}^{2}}{E x_{0}^{2}}\right)^{2}}, \\
H e_{a}=\left|E x_{0}^{1} \times E x_{0}^{2}\right| \sqrt{\left(\frac{H e_{0}^{1}}{E x_{0}^{1}}\right)^{2}+\left(\frac{H e_{0}^{2}}{E x_{0}^{2}}\right)^{2}},
\end{array}\right.
$$

and states $b, c$, and $d$ are calculated similarly.

(3) Organized CUGF:

$$
\begin{aligned}
U(Z)= & \left(E x_{a}, E n_{a}, H e_{a}\right) Z^{0}+\left(E x_{b}, E n_{b}, H e_{b}\right) Z^{0} \\
& +\left(E x_{c}, E n_{c}, H e_{c}\right) Z^{0}+\left(E x_{d}, E n_{d}, H e_{d}\right) Z^{1} .
\end{aligned}
$$

The digital characteristic parameters $E x_{0}, E n_{0}$, and $H e_{0}$ of the probability cloud of the MSS in state 0 are obtained as

$$
\left\{\begin{array}{l}
E x_{0}=E x_{a}+E x_{b}+E x_{c}, \\
E n_{0}=\sqrt{\left(E n_{a}\right)^{2}+\left(E n_{b}\right)^{2}+\left(E n_{c}\right)^{2}}, \\
H e_{0}=\sqrt{\left(H e_{a}\right)^{2}+\left(H e_{b}\right)^{2}+\left(H e_{c}\right)^{2}}
\end{array}\right.
$$

(4) CUGF of final MSS:

$$
U(Z)=C p_{0}\left(E x_{0}, E n_{0}, H e_{0}\right) Z^{0}+C p_{1}\left(E x_{1}, E n_{1}, H e_{1}\right) Z^{1} .
$$

When the MSS demand is $w$, the operation $\delta_{R}$ can be used to calculate the substitute reliability of the MSS. $\alpha_{i}$ is calculated by formula (7), and ( $E x, E n, H e$ ) by formula (16):

$$
\begin{aligned}
R(w) & =\delta_{R}\left(U^{\prime}(Z), w\right)=\delta_{R}\left(\sum_{i=1}^{k} C p_{i}(E x, E n, H e) z^{g_{i}}, w\right) \\
& =\sum_{i=1}^{k} C p_{i}(E x, E n, H e) \alpha_{i} .
\end{aligned}
$$

\section{CUGF of MSS under Common Cause Failure (CCF)}

4.1. CCF Failure Probability Cloud Calculation. Common causes of failure occurrences are the external environment, internal component aging, design and manufacturing quality, and human error. A common cause failure usually occurs when the load is greater than the working limit of the unit. In practical engineering, the unit is not directly ineffective but continues to exhibit degraded characteristics [58]. Assume that $V_{k}$ represents the severity of unit degradation, which is the probability of unit failure due to functional degradation. Parameter models that characterize a system 
TABle 1: Cloud operation rules.

\begin{tabular}{lccc}
\hline Symbol & $E x$ & $E n$ & $H e$ \\
\hline+ & $E x_{1}+E x_{2}$ & $\sqrt{E n_{1}^{2}+E n_{2}^{2}}$ & $\sqrt{H e_{1}^{2}+H e_{2}^{2}}$ \\
- & $E x_{1}-E x_{2}$ & $\sqrt{E n_{1}^{2}+E n_{2}^{2}}$ & $\sqrt{H e_{1}^{2}+H e_{2}^{2}}$ \\
$\times$ & $E x_{1} \times E x_{2}$ & $\left|E x_{1} E x_{2}\right| \sqrt{\left(E n_{1} / E x_{1}\right)^{2}+\left(E n_{2} / E x_{2}\right)^{2}}$ & $\left|E x_{1} E x_{2}\right| \sqrt{\left(H e_{1} / E x_{1}\right)^{2}+\left(H e_{2} / E x_{2}\right)^{2}}$ \\
$\div$ & $E x_{1} \div E x_{2}$ & $\left|\left(E x_{1} / E x_{2}\right)\right| \sqrt{\left(\left(E n_{1} / E x_{1}\right)\right)^{2}+\left(E n_{2} / E x_{2}\right)^{2}}$ & $\left|\left(E x_{1} / E x_{2}\right)\right| \sqrt{\left(H e_{1} / E x_{1}\right)^{2}+\left(H e_{2} / E x_{2}\right)^{2}}$ \\
\hline
\end{tabular}

due to common failure mainly include the $\alpha$ factor model, $\beta$ factor model, basic parameter (BP) model, multiple Greek letter (MGL) model, and square root model. The $\alpha$ factor model can be used to deal with common cause failure problems of any redundant system. It approximates the parameters in the model by the ratio of failure rates to suit the situation where there is less statistical data in the engineering system [59]. We use the weighted influence vector method and $\alpha$ factor model to estimate the common cause failure probability of a unit affected by both external environment and internal aging. However, in actual engineering, due to the complexity of the MSS structure, the external environmental factors in the course of operation are constantly changing, internal components are degraded, and there are errors in test data and expert experience. It has a fixed value, but it fluctuates within a certain range. We estimate $\alpha_{c}$ based on factors such as historical data, test data, physical characteristics, and expert experience. The distribution of sample $T$ is a cloud of common cause failure probability $\alpha_{c}$ and is called the common cause failure probability cloud.

4.2. CUGF under CCF Conditions. The fusion of common cause failure and CUGF is based on the following basic assumptions:

(1) The unit or system has a finite number of discrete states, each state is determined, and the system is unrepairable.

(2) The state probability of a unit or system has uncertainty.

(3) There are $n$ types of common cause failure groups in the system, each composed of the same unit. The generalized intensity of the unit is statistically independent.

(4) If the load is greater than the unit's endurance limit, or the unit is subjected to an external impact, the load on all units in the common cause group will exceed their strength limits, causing all components to fail simultaneously.

This paper discusses two failure modes of the common cause group: independent failure and simultaneous failure of all components of the common factor group. A CUGF with a common cause group can be expressed as

$$
U_{C}(Z)=\left(1-\alpha_{c}\right) U^{\prime}(Z)+\alpha_{c} Z^{x_{c}},
$$

where $\alpha_{c}$ is the common cause failure probability cloud, $U^{\prime}(Z)$ is the unit independent CUGF, and $x_{c}$ is the performance level of the common cause group when the common cause fails occurs.

\section{Case Analysis}

A fluid transfer MSS consists of three units. Subsystem 1 consists of units $A$ and $B$ in parallel and constitutes a common cause failure group. Subsystem 2 is connected in series with subsystem 1 by a $C$ unit. The MSS structure is shown in Figure 3. We assume that the state performance of each unit is an exact value, and the specific physical meaning of each unit's performance level is ignored. Units $A$ and $B$ each have three states $\{0,1,1.5\}$ and $\{0,1.5,2\}$, and unit $C$ has two states $\{0,4\}$.

5.1. MSS Reliability When the Unit Fails Independently. It is assumed that the state probability of each unit has 500 items of quantitative data or qualitative information. The state probability of units $A$ and $B$ comes from historical data, test data, and quantitative data. The state probability of unit $C$ comes from expert experience, field experience, and other qualitative information. The state probability of all information or data constitutes a sample set $X=\left\{x_{1}, x_{2}, \ldots, x_{500}\right\}$ and $Y=\left\{y_{1}, y_{2}, \ldots, y_{500}\right\}$. According to the algorithm in Section 2.2.2, we substitute the quantitative data from the sample sets of cells $A$ and $B$ in the inverse normal cloud generator to find the mean value $X$, center distance $M$, and variance $S^{2}$, so as to obtain the digital characteristic parameter value of the unit probability cloud. In accordance with the algorithm in Section 2.2.1, the cloud drops of the probability cloud of the unit are obtained by substituting them in the normal cloud generator, and the normal cloud map is drawn by MATLAB, reflecting the fuzziness and randomness of the state probability of each unit. Unit $C$ understands the qualitative information based on the experience of experts to obtain empirical digital characteristic parameter values. We obtain the numerical characteristic parameter values of the unit probability cloud according to formula (8). In accordance with the algorithm in Section 2.2.1, the cloud drops of the probability cloud of the unit are obtained by substituting them in the normal cloud generator, and the normal cloud map is drawn by MATLAB, reflecting the fuzziness and randomness of the state probability of each unit. Table 2 shows the numeric characteristic parameter values of the specific probability cloud, and normal cloud diagram is shown in Figures 4-6.

The CUGF of each unit of the MSS is obtained according to formulas (9) and (10) as follows: 


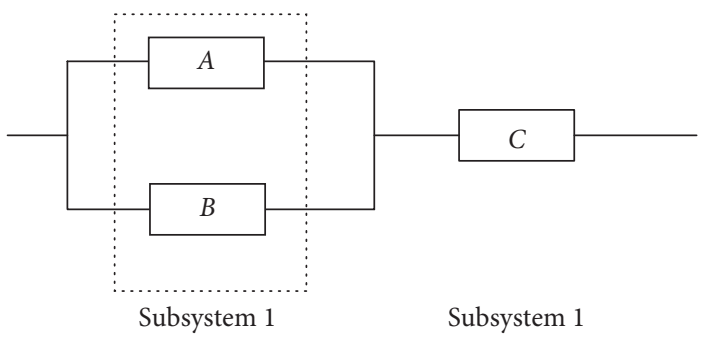

Figure 3: A fluid transmission system.

TABLE 2: Digital eigenvalue of cloud probability.

\begin{tabular}{lccc}
\hline Unit & $P_{1}^{j}$ & $P_{2}^{j}$ & $P_{3}^{j}$ \\
\hline$A$ & $\left(0.05996,0.0011,1.1720 e^{-4}\right)$ & $\left(0.12193,0.0039,8.8822 e^{-4}\right)$ & $\left(0.81811,0.0030,9.7399 e^{-4}\right)$ \\
$B$ & $\left(0.08995,0.0026,2.1219 e^{-4}\right)$ & $(0.24354,0.0048,0.0019)$ & $\left(0.6665,0.0035,7.0318 e^{-4}\right)$ \\
$C$ & $(0.07648,0.0159,0.00621)$ & $(0.92352,0.0159,0.00621)$ & \\
\hline
\end{tabular}

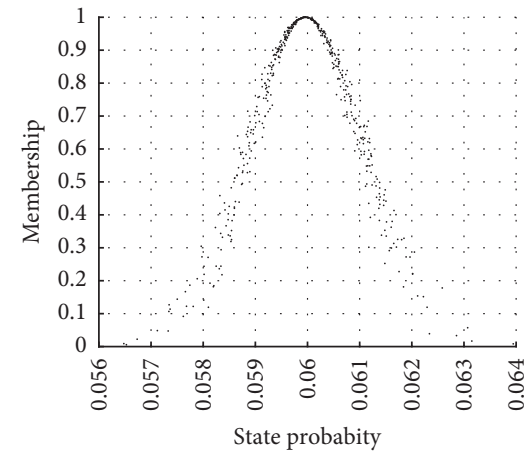

(a)

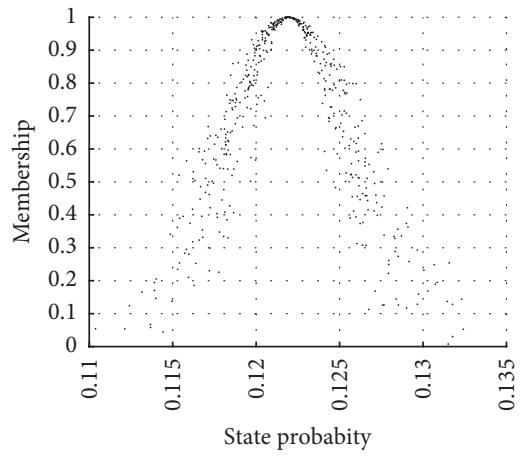

(b)

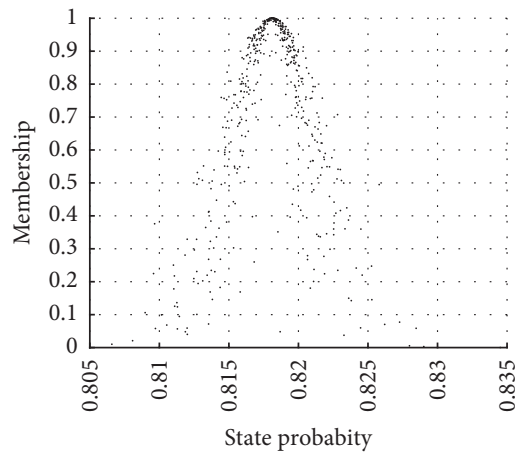

(c)

FIgURE 4: State probability normal cloud of unit $A$.

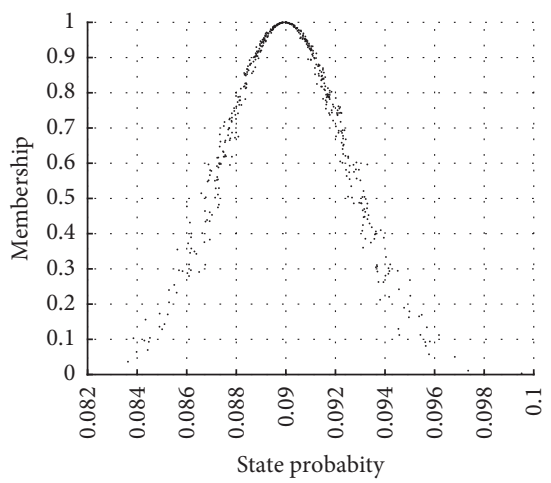

(a)

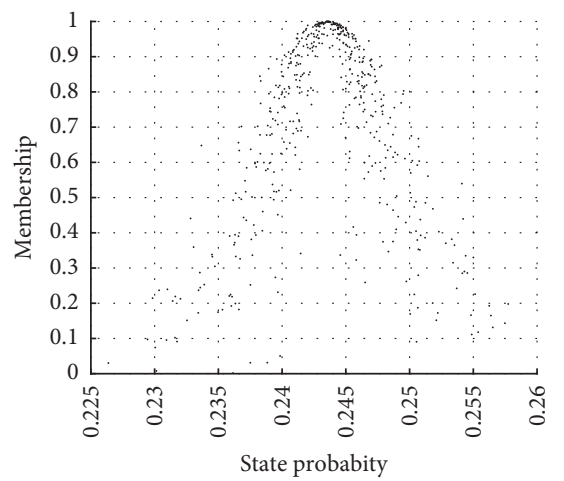

(b)

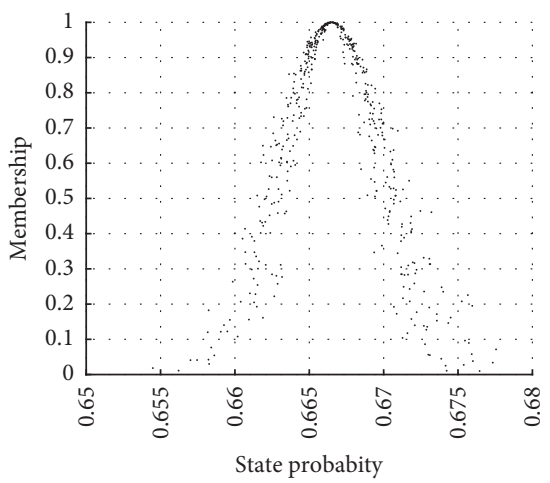

(c)

Figure 5: State probability normal cloud of unit $B$. 


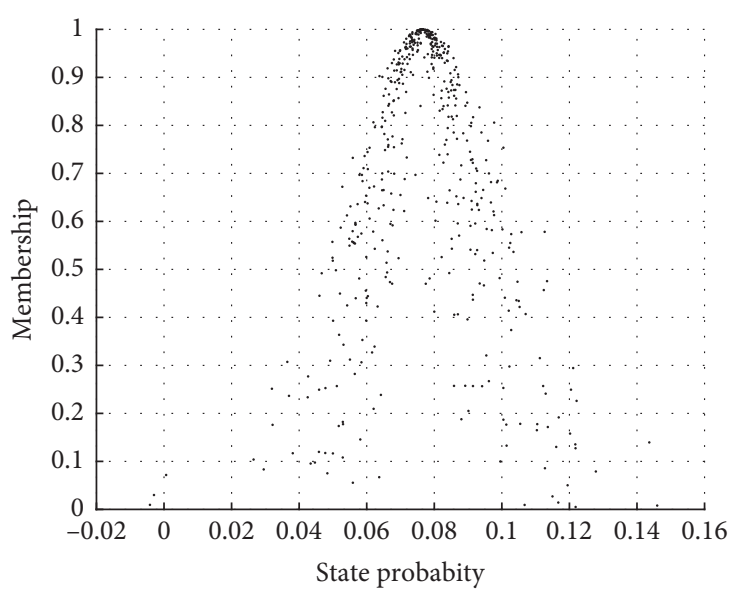

(a)

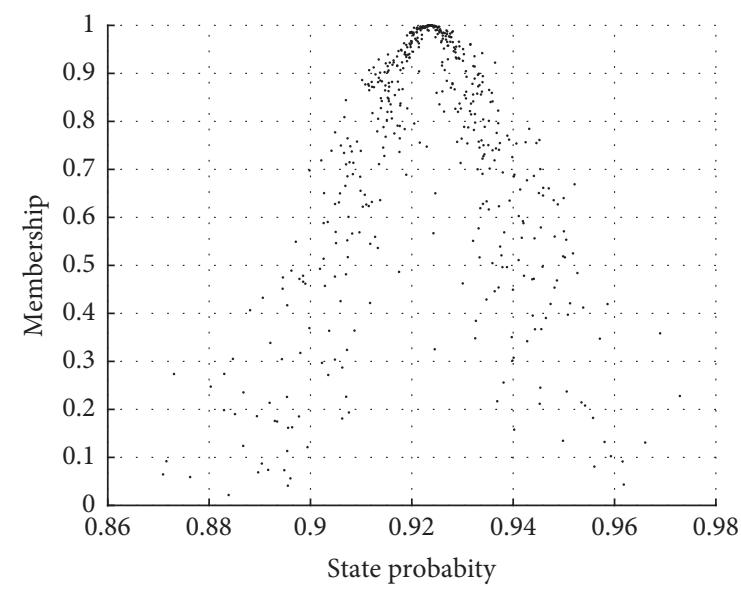

(b)

FiguRE 6: State probability normal cloud of unit $C$.

$$
\begin{aligned}
U^{\prime A}(Z)=\sum_{j=1}^{3} C p_{j}^{A}(E x, E n, H e) Z^{g_{j}^{A}}= & \left(0.05996,0.0011,1.1720 e^{-4}\right) Z^{0} \\
& +\left(0.12193,0.0039,8.8822 e^{-4}\right) Z^{1}+\left(0.81811,0.0030,9.7399 e^{-4}\right) Z^{1.5} \\
U^{\prime B}(Z)=\sum_{j=1}^{3} C p_{j}^{B}(E x, E n, H e) Z^{g_{j}^{B}}= & \left(0.08995,0.0026,2.1219 e^{-4}\right) Z^{0} \\
& +(0.24354,0.0048,0.0019) Z^{1.5}+\left(0.6665,0.0035,7.0318 e^{-4}\right) Z^{2} \\
U^{\prime C}(Z)=\sum_{j=1}^{2} C p_{j}^{C}(E x, E n, H e) Z^{g_{j}^{C}}= & (0.07648,0.0159,0.00621) Z^{0} \\
& +(0.92352,0.0159,0.00621) Z^{4}
\end{aligned}
$$

To facilitate calculation and programming, a matrix is used to represent each unit conversion function with multiple states, whose columns are, respectively, Ex, En, and $H e$. In the calculation process, for unit $j$ with $k_{j}$ states, the state probability cloud can be used as the matrix form of $k_{j} \times 3$ is expressed, and the result can be obtained through programming according to the defined two operation forms:

$$
\begin{aligned}
& U^{\prime A}(Z)=C p^{A} Z^{A}=\left[\begin{array}{lll}
0.05996 & 0.0011 & 1.1720 e^{-4} \\
0.12193 & 0.0039 & 8.8822 e^{-4} \\
0.81811 & 0.0030 & 9.7399 e^{-4}
\end{array}\right] \cdot\left[\begin{array}{c}
Z^{0} \\
Z^{1} \\
Z^{1.5}
\end{array}\right]^{T}, \\
& U^{\prime B}(Z)=C p^{B} Z^{B}=\left[\begin{array}{lll}
0.08995 & 0.0026 & 2.1219 e^{-4} \\
0.24354 & 0.0048 & 0.0019 \\
0.6665 & 0.0035 & 7.0318 e^{-4}
\end{array}\right] \cdot\left[\begin{array}{c}
Z^{0} \\
Z^{1.5} \\
Z^{2}
\end{array}\right]^{T}, \\
& U^{\prime C}(Z)=C p^{C} Z^{C}=\left[\begin{array}{lll}
0.07648 & 0.0159 & 0.00621 \\
0.92352 & 0.0159 & 0.00621
\end{array}\right] \cdot\left[\begin{array}{c}
Z^{0} \\
Z^{4}
\end{array}\right]^{T} .
\end{aligned}
$$

According to the structural relationship of MSS in Figure 3 and the arithmetic operators $\Phi_{P}$ and $\Phi_{S}$ of the structural relationship of formulas (3) and (4), the CUGF of subsystem 1 and MSS is obtained. The operations between 
the matrices use specific operations based on the operation rules defined above. According to formulas (14) and (16), the matrix form is obtained as

$$
\begin{aligned}
\Omega_{\phi_{S}}=\left(U^{\prime A}(Z), U^{\prime B}(Z)\right) & =\left[\begin{array}{ccc}
0.00539 & 0.00018 & 1.6513 e^{-5} \\
0.01098 & 0.0004 & 8.3559 e^{-5} \\
0.08819 & 0.00214 & 0.00022 \\
0.03996 & 0.00076 & 8.8759 e^{-5} \\
0.02969 & 0.0011 & 0.00032 \\
0.28052 & 0.0047 & 0.00168 \\
0.54527 & 0.0035 & 0.00087
\end{array}\right] \cdot\left[\begin{array}{c}
Z^{0} \\
Z^{1} \\
Z^{1.5} \\
Z^{2} \\
Z^{2.5} \\
Z^{3} \\
Z^{3.5}
\end{array}\right]^{T}, \\
U^{\prime}(Z)=\Omega_{\phi p}\left(\Omega_{\phi_{S}}\left(U^{\prime A}(Z), U^{\prime B}(Z)\right), U^{\prime C}(Z)\right) & =\left[\begin{array}{lll}
0.08145 & 0.0099 & 0.0039 \\
0.01014 & 0.00041 & 0.00010 \\
0.08145 & 0.00242 & 0.00058 \\
0.03690 & 0.00095 & 0.00026 \\
0.02742 & 0.00112 & 0.00035 \\
0.25907 & 0.00622 & 0.00233 \\
0.50357 & 0.00925 & 0.00348
\end{array}\right] \cdot\left[\begin{array}{c}
Z^{0} \\
Z^{1} \\
Z^{1.5} \\
Z^{2} \\
Z^{2.5} \\
Z^{3} \\
Z^{3.5}
\end{array}\right]^{T} .
\end{aligned}
$$

When the MSS performance demand level is $w=1.5$, according to formulas (9), (18), and (16), the reliability of the MSS in the case of independent failure is obtained as

$$
R_{w}=\delta_{R}\left(U^{\prime}(Z), 1.5\right)=(0.90841,0.0115,0.0043) .
$$

According to the forward normal cloud generator algorithm in Section 2.2.1, the reliability normal cloud diagram of MSS independent failure is obtained using MATLAB, as shown in Figure 7. The normal cloud diagram reflects the mixed uncertainty of reliability in the case of MSS independent failure.

5.2. MSS Reliability with Consideration of Common Cause Failures. The $\alpha_{c}$ sampled by the method of [58] formed a sample consisting of quantitative data. The sample has a capacity of 100 , which constitutes a sample set $T=\left\{t_{1}, t_{2}, \ldots, t_{100}\right\}$ according to the algorithm in Sections 2.2.1 and 2.2.2. We find the digital characteristic parameter value of the common cause probability cloud $\alpha_{c}=\left(0.1308,0.0040,7.6070 e^{-4}\right)$. The cloud drop and the normal cloud diagram of the common cause failure probability cloud are shown in Figure 8.

Subsystem 1 formed by units $A$ and $B$ forms a common cause failure group. Therefore, according to formulas (19), (14), and (16), the corresponding CUGF and the operations between the matrices use specific operations based on the operation rules defined above. Application matrix forms of subsystem 1 can be obtained as

$$
\begin{aligned}
U_{C}^{A, B}(Z)= & \left(1-\alpha_{C}\right)\left(U_{A, B}^{\prime}(Z)\right)+\alpha_{C} Z^{x_{c}}=\left(0.8692,0.0040,7.6070 e^{-4}\right)\left(\left(0.00539,0.00018,1.6513 e^{-5}\right) Z^{0}\right. \\
& +\left(0.01098,0.0004,8.3559 e^{-5}\right) Z^{1}+(0.08819,0.00214,0.00022) Z^{1.5}+\left(0.03996,0.00076,8.8759 e^{-5}\right) Z^{2} \\
& \left.+(0.02969,0.0011,0.00032) Z^{2.5}+(0.28052,0.0047,0.00168) Z^{3}+(0.54527,0.0035,0.00087) Z^{3.5}\right) \\
& +\left(0.1308,0.0040,7.6070 e^{-4}\right) Z^{0}=(0.13548,0.00400,0.00077) Z^{0}+\left(0.00954,0.00035,7.3079 e^{-5}\right) Z^{1} \\
& +(0.07665,0.00189,0.00020) Z^{1.5}+\left(0.03473,0.00068,8.2914 e^{-5}\right) Z^{2}+(0.02581,0.00096,0.00028) Z^{2.5} \\
& +(0.24383,0.00424,0.00148) Z^{3}+(0.47396,0.00374,0.00086) Z^{3.5} \\
U_{C}^{A, B}(Z)= & {\left[\begin{array}{lll}
0.13548 & 0.00400 & 0.00077 \\
0.00954 & 0.00035 & 7.3079 e^{-5} \\
0.07665 & 0.00189 & 0.00020 \\
0.03473 & 0.00068 & 8.2914 e^{-5} \\
0.02581 & 0.00096 & 0.00028 \\
0.24383 & 0.00424 & 0.00148 \\
0.47396 & 0.00374 & 0.00086
\end{array}\right] \cdot\left[\begin{array}{c}
Z^{0} \\
Z^{1} \\
Z^{1.5} \\
Z^{2} \\
Z^{2.5} \\
Z^{3} \\
Z^{3.5}
\end{array}\right] . }
\end{aligned}
$$




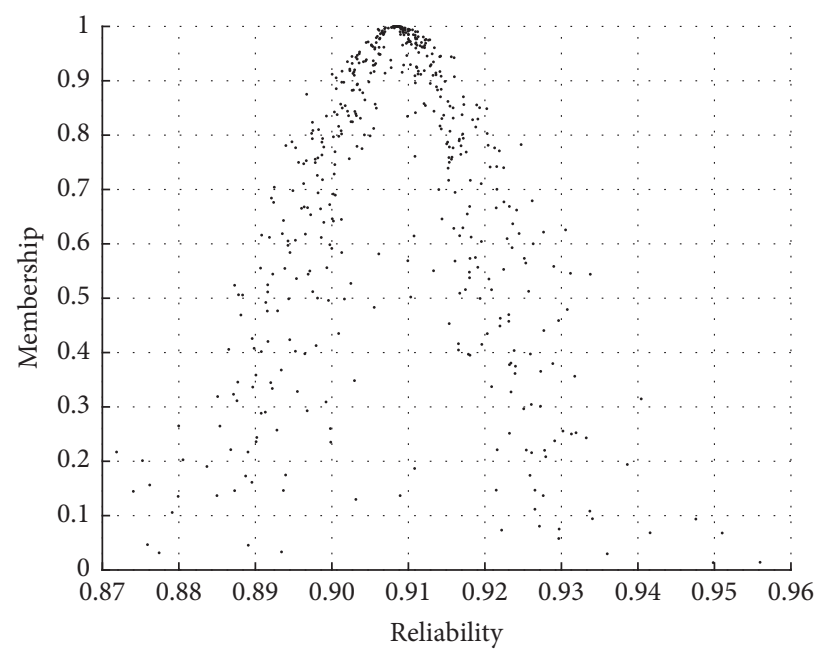

FIgURE 7: MSS independent failure reliability true cloud.

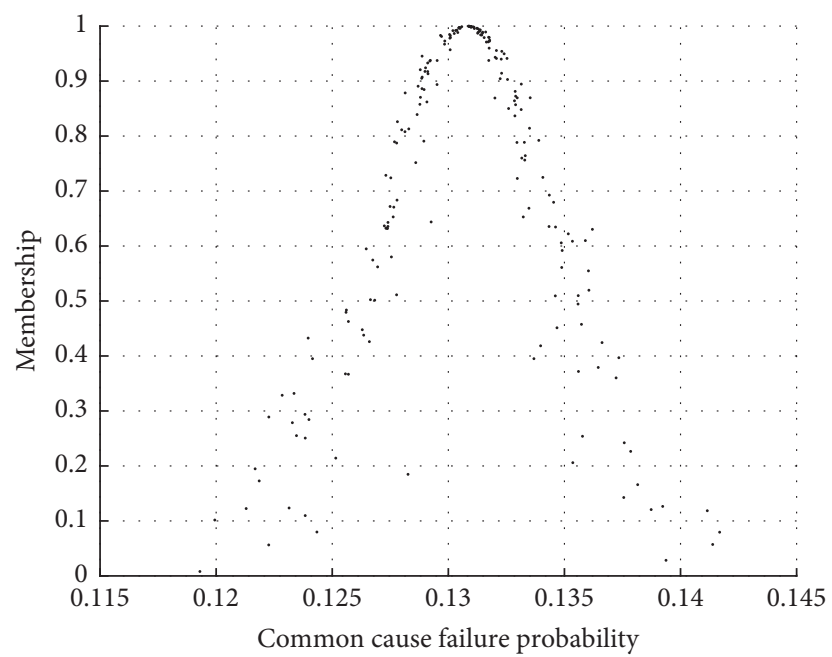

FIGURE 8: Common cause failure probability cloud.

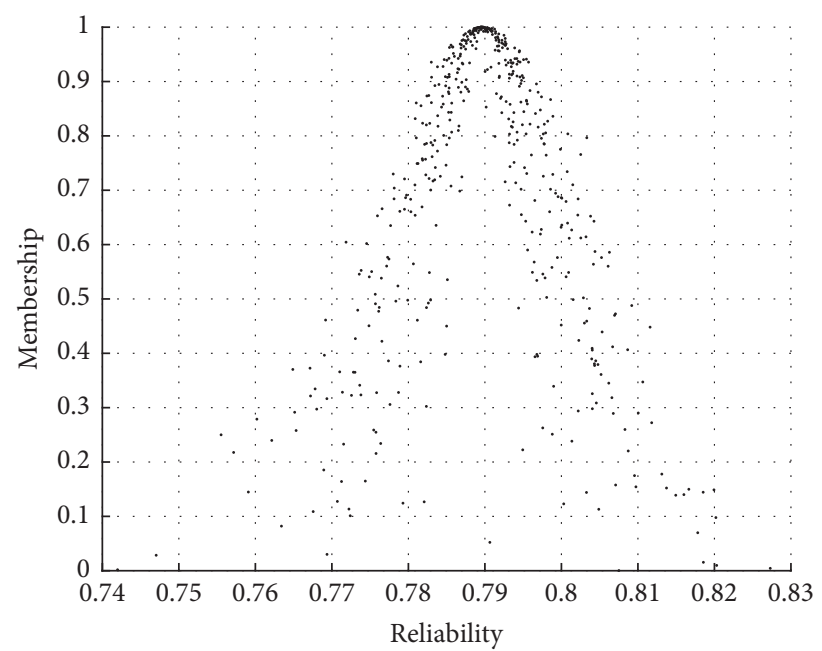

FIGURE 9: MSS common cause failure reliability true cloud. 


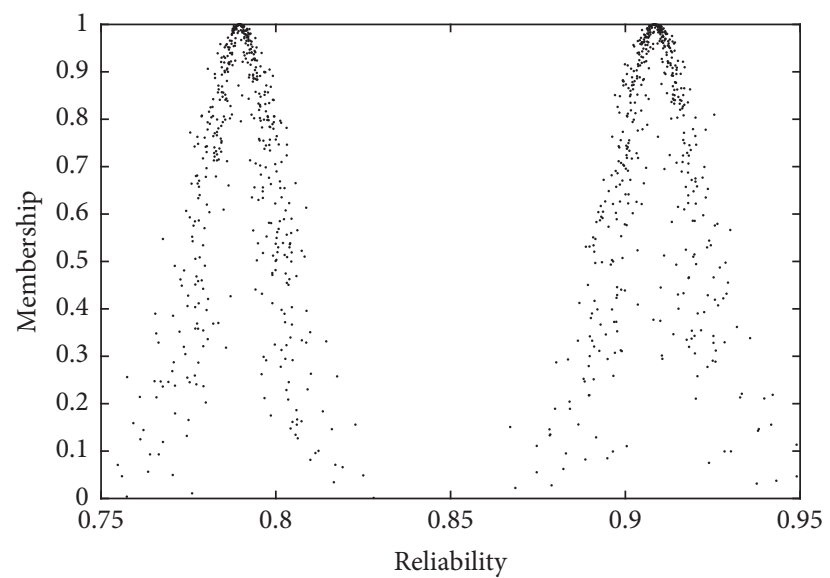

FIGURE 10: MSS independent failure and common cause failure comparison reliability normal cloud.

The CUGF of the MSS when the common cause fails is

$$
\begin{aligned}
U_{C}(Z)= & \Omega_{\phi p}\left(U_{C}^{A, B}(Z), U^{\prime C}(Z)\right)=(0.2016,0.0099,0.0036) Z^{0} \\
& +\left(0.00881,0.00036,8.9800 e^{-5}\right) Z^{1}+(0.07079,0.00213,0.00051) Z^{1.5} \\
& +(0.03207,0.00084,0.00023) Z^{2}+(0.02384,0.00098,0.00030) Z^{2.5} \\
& +(0.22518,0.00551,0.00204) Z^{3}+(0.43771,0.00829,0.00305) Z^{3.5}
\end{aligned}
$$

We can write this in matrix form as

$$
U_{C}(Z)=\Omega_{\phi p}\left(U_{C}^{A, B}(Z), U^{\prime C}(Z)\right)=\left[\begin{array}{ccc}
0.2016 & 0.0099 & 0.0036 \\
0.00881 & 0.00036 & 8.9800 e^{-5} \\
0.07079 & 0.00213 & 0.00051 \\
0.03207 & 0.00084 & 0.00023 \\
0.02384 & 0.00098 & 0.00030 \\
0.22518 & 0.00551 & 0.00204 \\
0.43771 & 0.00829 & 0.00305
\end{array}\right] \cdot\left[\begin{array}{c}
Z^{0} \\
Z^{1} \\
Z^{1.5} \\
Z^{2} \\
Z^{2.5} \\
Z^{3} \\
Z^{3.5}
\end{array}\right]^{T}
$$

When the MSS performance requirement level is $w=1.5$, according to the cloud algorithm of formulas (7), (19), and (16), the reliability of the MSS under common cause failure is obtained as

$$
R(w)=\delta_{R}\left(U_{C}(Z), 1.5\right)=(0.78959,0.0103,0.0037) .
$$

According to the forward normal cloud generation algorithm in Section 2.2.1, the normal cloud diagram of reliability when MSS common cause failure is obtained is shown in Figure 9. The normal cloud diagram reflects the mixed uncertainty of the reliability of MSS common cause failure.
5.3. Comparative Analysis. Comparing the reliability of the MSS between independent failure and common cause failure, it can be seen from Figure 10 that the reliability in the case of common cause failure is lower than the reliability of independent failure of the MSS unit. The MSS reliability expectation decreased by 0.11882 due to common cause failure. The reliability calculated by ignoring common cause failure will have a large error. It is necessary to consider the common cause failure condition, so that it is more consistent with the actual project. Therefore, in actual engineering, the ultimate strength of the unit should be improved, and corresponding protection measures should be taken for a 
unit that is prone to common cause failure, thus reducing its occurrence. The uncertainty of the reliability of the MSS unit in the case of independent failure and common cause failure is basically the same; i.e., the difference between the entropy value and super entropy value is not large. The uncertainty of the common cause failure rate has little effect on the uncertainty of MSS reliability.

\section{Conclusion}

This paper addresses the reliability analysis problem of MSS unit state probability information with mixed uncertainty under common cause failure. Based on a probabilistic cloud model, the traditional universal generation function is extended to obtain CUGF. Using the cloud model to represent the uncertainty of the probability information of each state of the unit, the CUGF is used to analyze the reliability of the unit state probabilistic information with mixed uncertainty under independent failure and common cause failure. The system's multiple states, information uncertainty, and common cause failure are well integrated. In this way, the reliability of the analysis is more accurate, and large errors are avoided in engineering practice. The next step is to consider the reliability analysis of MSS units and subsystems using CUGF when the performance state and state probability are both uncertain. The cloud model and information fusion theory are combined to effectively deal with the situation where the unit state probability information has mixed uncertainties.

\section{Data Availability}

The data used to support the findings of this study are available from the corresponding author upon request.These data include historical data, test data, expert experience, and usage experience.

\section{Conflicts of Interest}

The authors declare that there are no conflicts of interest regarding the publication of this article.

\section{Acknowledgments}

The authors thank LetPub (http://www.letpub.com) for its linguistic assistance during the preparation of this manuscript. They are also grateful to National Natural Science Foundation of China (51374121) and Liaoning Distinguished Professor Funding Project (551710007007).

\section{References}

[1] R. M. Barton and W. W. Damon, "Reliability in a multi-state system," in Proceedings of the Sixth Annual Southeastern Symposium on Systems Theory, Baton Rouge, LA, USA, October 1974.

[2] J. D. Murchland, "Fundamental concepts and relations for 0 reliability analysis of multi-state systems and fault tree analysis," Theoretical and Applied Aspects of System Reliability and Fault Tree Analysis, pp. 581-618 SIAM, Philadelphia, PA, USA, 1975.
[3] R. E. Barlow and A. S. Wu, "Coherent systems with multi-state components," Mathematics of Operations Research, vol. 3, no. 4, pp. 275-281, 1978.

[4] E. El-Neveihi, F. Prochan, and J. Setharaman, "Multi-state coherent systems," Journal of Applied Probability, vol. 15, pp. 675-688, 1978.

[5] K. Krzysztof, S. Joanna, J. Miroslaw, and D. Przemyslaw, "On multi-state safety analysis in shipping," International Journal of Reliability, Quality and Safety Engineering, vol. 14, no. 6, pp. 547-567, 2007.

[6] K. Kolowrocki and B. Kwiatuszewska-sarnecka, "Reliability and risk analysis of large systems with ageing components," Reliability Engineering and System Safety, vol. 93, pp. 18211829, 2008.

[7] C. Dongning and Y. Chengyu, "Reliability analysis of polymorphic system based on fuzzy bayesian network and its application in hydraulic system," Journal of Mechanical Engineering, vol. 48, no. 16, pp. 175-183, 2012.

[8] L. Yu, L. Xiangyu, and Z. Xiaohu, "Reliability modeling and optimization of multi-State system considering load dynamic allocation mechanism," Journal of Mechanical Engineering, vol. 52, no. 6, pp. 197-205, 2016.

[9] Y. Massim, A. Zeblah, R. Meziane, M. Benguediab, and A. Ghouraf, "Optimal design and reliability evaluation of multi-state series-parallel power systems," Nonlinear Dynamics, vol. 40, no. 4, pp. 309-321, 2005.

[10] Y. Massim, A. Zeblah, M. Benguediab, A. Ghouraf, and R. Meziane, "Reliability evaluation of electrical power systems including multi-state considerations," Electrical Engineering, vol. 88, no. 2, pp. 109-116, 2006.

[11] H. A. Taboada, J. F. Espiritu, and D. W. Coit, "Design allocation of multistate series-parallel systems for power systems planning: a multiple objective evolutionary approach," Proceedings of the Institution of Mechanical Engineers, Part O: Journal of Risk and Reliability, vol. 222, no. 3, pp. 381-391, 2008.

[12] Y.-F. Li and E. Zio, "A multi-state model for the reliability assessment of a distributed generation system via universal generating function," Reliability Engineering and System Safety, vol. 106, pp. 28-36, 2012.

[13] J. F. Caete and J. H. Saleh, "Beyond reliability multi-state failure analysis of satellite subsystems: A statistical approach," Reliability Engineering and System Safety, vol. 95, no. 4, pp. 311-322, 2010.

[14] S. Y. Kim, J.-F. Castet, and J. H. Saleh, "Spacecraft electrical power subsystem: failure behavior, reliability, and multi-state failure analyses," Reliability Engineering and System Safety, vol. 98, no. 1, pp. 55-65, 2012.

[15] J. K. Wayer, J.-F. Castet, and J. H. Saleh, "Spacecraft attitude control subsystem: reliability, multi-state analyses, and comparative failure behavior in LEO and GEO," Acta Astronautica, vol. 85, pp. 83-92, 2013.

[16] W.-C. Yeh, "A simple universal generating function method for estimating the reliability of general multi-state node networks," IIE Transactions, vol. 41, no. 1, pp. 3-11, 2008.

[17] C. C. Jane and Y. W. Laih, "A practical algorithm for computing multi-state two-terminal reliability," IEEE Transactions on Reliability, vol. 57, no. 2, pp. 295-302, 2008.

[18] H. Ning and W. Zhiwei, "Overview of network reliability assessment models and algorithms," Systems Engineering and Electronics, vol. 35, no. 12, pp. 2651-2657, 2013.

[19] C. Gianni, G. Emilio, P. Fabrizio et al., "Reliability assessment in smart distribution networks," Electric Power Systems Research, vol. 104, pp. 164-175, 2013. 
[20] A. Lisnianski and G. Levitin, Multi-State System Reliability: Assessment, Optimization and Applications, World Scientific Publishing Co Pte Ltd, New York, NY, USA, 2003.

[21] E. Zaitseva, S. Kovalik, V. Levashenko et al., "Algorithm for dynamic analysis of multi-state system by structure function," in Proceedings of the IEEE International Conference on Computer as a Tool (EUROCON 2005), pp. 1224-1227, Belgrade, Serbia, November 2005.

[22] E. Zaitseva and V. Levashenko, "Investigation multi-state system reliability by structure function," in Proceedings of the 2nd International Conference on Dependability of Computer Systems, pp. 81-90, Szklarska Poreba, Poland, 2007.

[23] Z. Zhongbao, M. Chaoqun, Z. Jinglun et al., "Polymorphic fault tree analysis method based on bayesian network," Mathematics in Practice and Theory, vol. 38, no. 19, pp. 89-95, 2008.

[24] A. Lisnianski, "Extended block diagram method for a multistate system reliability assessment," Reliability Engineering and System Safety, vol. 92, no. 12, pp. 1601-1607, 2007.

[25] I. W. Soro, M. Nourelfath, and D. Aït-Kadi, "Performance evaluation of multi-state degraded systems with minimal repairs and imperfect preventive maintenance," Reliability Engineering and System Safety, vol. 95, no. 2, pp. 65-69, 2010.

[26] A. Lisnianski, D. Elmakias, D. Laredo, and H. Ben Haim, “A multi-state Markov model for a short-term reliability analysis of a power generating unit," Reliability Engineering and System Safety, vol. 98, no. 1, pp. 1-6, 2012.

[27] J. E. Ramirez-marquez and D. W. Coit, "A Monte-Carlo simulation approach for approximating multi-state two-terminal reliability," Reliability Engineering and System Safety, vol. 87, no. 2, pp. 253-264, 2005.

[28] E. Zio, M. Marella, and L. Podofillini, "A Monte Carlo simulation approach to the availability assessment of multistate systems with operational dependencies," Reliability Engineering and System Safety, vol. 92, no. 7, pp. 871-882, 2007.

[29] H. Fan and X. Sun, "A multi-state reliability evaluation model for P2P networks," Reliability Engineering and System Safety, vol. 95, no. 4, pp. 402-411, 2010.

[30] G. Levitin, The Universal Generating Function in Reliability Analysis and Optimization, Springer, London, UK, 2005.

[31] G. Levitin and L. Xing, "Reliability and performance of multistate systems with propagated failures having selective effect," Reliability Engineering and System Safety, vol. 95, no. 6, pp. 655-661, 2010.

[32] G. Levitin, L. Liudong Xing, H. Ben-Haim, and Y. Dai, "Reliability of series-parallel systems with random failure propagation time," IEEE Transactions on Reliability, vol. 62, no. 3, pp. 637-647, 2013.

[33] I. Ushakov, "Universal generating function," Soviet Journal of Computer and Systems Sciences, vol. 24, no. 5, pp. 118-129, 1986.

[34] I. Ushakov, "Optimal standby problems and a universal generating function," Soviet Journal of Computer and Systems Sciences, vol. 25, no. 4, pp. 79-82, 1987.

[35] Y. Ding and A. Lisnianski, "Fuzzy universal generating functions for multi-state system reliability assessment," Fuzzy Sets and Systems, vol. 159, no. 3, pp. 307-324, 2008.

[36] J. Mula, R. Poler, and J. P. Garcia-Sabater, "Material requirement planning with fuzzy constraints and fuzzy coefficients," Fuzzy Sets and Systems, vol. 158, no. 7, pp. 783-793, 2007.

[37] S. Sankararaman and S. Mahadevan, "Likelihood-based representation of epistemic uncertainty due to sparse point data and/or interval data," Reliability Engineering and System Safety, vol. 96, no. 7, pp. 814-824, 2011.

[38] G. Curcurù, G. M. Galante, and C. M. La Fata, "Epistemic uncertainty in fault tree analysis approached by the evidence theory," Journal of Loss Prevention in The Process Industries, vol. 25, no. 4, pp. 667-676, 2012.

[39] Z. Zhang, C. Jiang, G. G. Wang et al., "An efficient reliability analysis method for structures with epistemic uncertainty using evidence theory," in Proceedings of the ASME 2014 International Design Engineering Technical Conferences and Computers and Information in Engineering Conference, New York, NY, USA, 2014.

[40] Y. Li and E. Zio, "Uncertainty Analysis of the adequacy assessment model of a distributed generation system," Renewable Energy, vol. 41, no. 1, pp. 235-244, 2012.

[41] M. Drouin, Guidance on the Treatment of Uncertainties Associated with PRAs in Risk-Informed Decision Making: Main Report, US: Nuclear Regulatory Commission, Rockville, MD, USA, 2009.

[42] Y. Ding, M. J. Zuo, A. Lisnianski, and Z. Tian, "Fuzzy multistate systems: general definitions, and performance assessment," IEEE Transactions on Reliability, vol. 57, no. 4, pp. 589-594, 2008.

[43] C.-y. Li, X. Chen, X.-s. Yi, and J.-y. Tao, "Interval-valued reliability analysis of multi-state systems," IEEE Transactions on Reliability, vol. 60, no. 1, pp. 323-330, 2011.

[44] S. Destercke and M. Sallak, "An extension of universal generating function in multi-state systems considering epistemic uncertainties," IEEE Transactions on Reliability, vol. 62, no. 2, pp. 504-514, 2013.

[45] G. Long, S. Tailiang, and Y. Xu, "Multi-state system reliability assessment based on cloud model," Journal of Academy of Armored Forces Engineering, vol. 30, no. 5, pp. 100-106, 2016.

[46] L. Deyi and L. Changwei, "On the universality of the normal cloud model," China Engineering Science, vol. 6, no. 8, pp. 28-34, 2004.

[47] L. Deyi and D. Fu, Uncertain Artificial Intelligence, pp. 229-230, National Defense Industry Press, Beijing, China, 2005.

[48] G. Levitin, "Incorporating common-cause failures into nonrepairable multistate series-parallel system analysis," IEEE Transactions on Reliability, vol. 50, no. 4, pp. 380-388, 2001.

[49] Z. Jinyu, X. Liyang, and W. Xuemin, "Common cause failure analysis and reliability model for multi-state systems," Journal of Mechanical Engineering, vol. 41, no. 6, pp. 66-70, 2005.

[50] G. Levitin, "Common supply failures in linear multi-state sliding window systems," Reliability Engineering and System Safety, vol. 82, no. 1, pp. 55-62, 2003.

[51] Z. Jinyu and X. Liyang, "Common cause failure mechanism and quantitative analysis of multi-state systems," Journal of Mechanical Engineering, vol. 44, no. 10, pp. 77-82, 2008.

[52] L. Chunyang, C. Xun, and Y. Xiaoshan, "Reliability optimization of polymorphic systems considering common cause failure," China Mechanical Engineering, vol. 21, no. 2, pp. 155-159, 2010.

[53] J. Mi, Y.-F. Li, Y. Liu, Y.-J. Yang, and H.-Z. Huang, "Belief universal generating function analysis of multi-state systems under epistemic uncertainty and common cause failures," IEEE Transactions on Reliability, vol. 64, no. 4, pp. 1300-1309, 2015.

[54] C. Y. Liu, M. Feng, X. J. Dai et al., "A new algorithm of backward cloud," Journal of System Simulation, vol. 16, no. 11, pp. 2417-2420, 2004. 
[55] Q. Yi, L. Qiang, and H. Sitan, "Research on system performance evaluation method based on cloud model and information fusion theory," Computer Engineering and Science, vol. 34, no. 2, pp. 181-185, 2012.

[56] G. Qiang and B. Yiming, "Effectiveness evaluation of missile information operation command based on cloud model," Command and Control and Simulation, vol. 30, no. 4, pp. 61-64, 2008.

[57] Y. B. Shi, H. Liu, and J. Sun, "The cloud model based on grey system theory and application on effectiveness evaluation," Intelligent Computing Theories, pp. 31-38, Springer, Nanning, China, 2013.

[58] W. Wei, X. Weidong, and W. Hong, "Research on common cause failure analysis method of safety instrument system based on differentiated design technology," China Safety Science Journal, vol. 23, no. 3, pp. 91-96, 2013.

[59] NUREG/CR-6268, Rev1_CCF Database and Analysis System Event Data Collection, Classification, and Coding, U.S. Nuclear Regulatory Commission, Washington, DC, USA, 2007. 